\title{
Wave-front conversion between a Gaussian beam with a cylindrical phase function and a plane wave in a monomode on-axis transmission holographic coupler
}

\author{
Pavel Cheben* and María L. Calvo \\ Department of Optics, Faculty of Physics, Complutense University, Ciudad Universitaria, Madrid 28040, Spain
}

Received January 4, 1993; revised manuscript accepted June 16, 1993; accepted June 21, 1993

\begin{abstract}
We present a coupled-wave-analysis-based treatment for a wave-front conversion problem between a Gaussian beam with a cylindrical phase and a plane wave for a two-dimensional on-axis geometry. A coupled-wave approach to the multiple-scattering process in an inhomogeneous dielectric medium offers a clear physically interpretable description of the diffraction phenomena in volume media as well as the possibility of an intelligible mathematical treatment. An analytical integral solution for the amplitudes of the two modes of diffraction is submitted. High diffraction efficiency $(\approx 1)$ and the deterioration of reconstruction fidelity are predicted. It is of interest to apply the model to the analysis and design of a monomode holographic fiber-to-fiber coupler, wave-front correctors, and holographic interconnection devices.
\end{abstract}

\section{INTRODUCTION}

The development of applications of monomode optical communication and interconnection systems during the past several years reflects the need to accommodate a variety of previously multimode optoelectronic elements to a single-mode regime.

In the case of holographic couplers, a majority of existing theoretical models for wave-front conversion in volume gratings could easily be used to describe a monomode coupling situation. However, as will be shown in this paper, some new theoretical aspects appear in the analysis of monomode holocouplers. Both practical and theoretical problems arise from the low numerical aperture of monomode fibers, and these problems generally lead to two trends: (1) the decrease of active conversion area and (2) the increased fiber-holographic-medium separation. The first concept is limited by the required minimum signalto-noise ratio, which decreases simultaneously with the decrease in recording area that is due to the scattering process in the volume medium. The decrease of apparent grain size of holographic media by antiscatter dyes can partially compensate the drop in the signal-to-noise ratio, but it increases losses significantly at recording. Even stronger limitations are due to the restrictions that overlapping (finite-beam) geometry and the associated Borrmann-like effect ${ }^{1-6}$ impose on the fidelity of reconstruction. The optimal solution seems to be offered by the increased fiber-holographic-media separation; however, this produces a situation in which the phase function of the Gaussian beam emerging from the optical fiber has a spherical (cylindrical in two-dimensional geometry) character in the space of the recording medium, and it is not realistic to continue with the plane phase approximation. Until now there was no theoretical model in the literature for describing the conversion between a Gaussian beam with spherical phase and a plane wave. Our research submits an analytical integral solution for the aforementioned problem in two-dimensional, on-axis, transmission geometry.
Classic works, treating the diffraction of a Gaussian beam in volume media ${ }^{7-11}$ have assumed the plane character of the phase function. Parry and Solymar ${ }^{12}$ offer a general solution for the volume holograms with twodimensional reducible geometry by using the generalized series method. It is difficult, nevertheless, to apply this method in complicated geometries because of its slow convergence and extraordinarily compounded analytical expression for higher-order terms of the series. The spherical- or cylindrical-to-plane-wave conversion is also analyzed in many studies. Guibelalde and Calvo's clear and straightforward method ${ }^{13}$ based on generalized threedimensional coordinates and permitting solution of the wave equation by separation of variables is of considerable interest. However, once again, it assumes a classic spherical-to-plane-wave conversion, and it does not involve Gaussian amplitude in the analysis. In addition, diffraction modes are not normalized, and the amplitude function of the -first mode is not consistent with other models ${ }^{14,15}$ or with our experimental results. ${ }^{16}$

\section{THEORETICAL MODEL}

The proposed model is based on a coupled-wave-analysis approach ${ }^{17}$ to the diffraction problem in volume gratings. The complex process for deriving the coupled-wave equations for the structure emerging from a generalized recording process may be found in detail in the book by Solymar and Cooke. ${ }^{18}$ However, some fundamental concepts necessary for an understanding of the problem will be presented here.

\section{Recording Process}

Let us assume an equal, generally complex value for the permittivity of the incidence and holographic media. In other words, the reflection and refraction effects are ignored. It is also assumed that both media are nonmagnetic $\left(\mu_{r}=1\right)$.

The two recording waves $\mathbf{E}_{10}$ and $\mathbf{E}_{20}$ interfere in the holographic medium area with their electric-field vectors: 


$$
\begin{aligned}
\mathbf{E}_{10}(x, y, t) & =\Psi_{10}(x, y) \phi_{10}(x, y) \exp \left[-\gamma_{0} p_{10}(x, y)+j \omega t\right] \hat{u}_{z} \\
& =\Omega_{10}(x, y) \exp \left[-\gamma_{0} p_{10}(x, y)+j \omega t\right] \hat{u}_{z}, \\
\mathbf{E}_{20}(x, y, t) & =\Psi_{20}(x, y) \phi_{20}(x, y) \exp \left[-\gamma_{0} p_{20}(x, y)+j \omega t\right] \hat{u}_{z} \\
& =\Omega_{20}(x, y) \exp \left[-\gamma_{0} p_{20}(x, y)+j \omega t\right] \hat{u}_{z},
\end{aligned}
$$

where $\gamma_{0}=j \beta_{0}+\alpha_{0}$ is a wave-propagation constant in an unexposed holographic medium where the interference between $\mathbf{E}_{10}$ and $\mathbf{E}_{20}$ occurs. As was assumed above, the medium is nonmagnetic with a complex value of the permittivity $\varepsilon_{r 0}=\varepsilon_{r 0}{ }^{\prime}-j \varepsilon_{r 0}{ }^{\prime \prime}$. The absorption constant $\alpha_{0}$ could, following directly from Maxwell's equations, link with the imaginary part $\beta_{0}$ of the propagation constant by the relation $\alpha_{0}=\beta_{0} \varepsilon_{r 0}{ }^{\prime \prime} /\left(2 \varepsilon_{r 0}{ }^{\prime}\right) . \quad \Psi_{10}(x, y), \Psi_{20}(x, y)$, $\phi_{10}(x, y), \phi_{20}(x, y)$ are scalar amplitudes, $p_{10}(x, y), p_{20}(x, y)$ are the phase functions, and $\hat{u}_{z}$ is a unit vector perpendicular to the plane of incidence (as can be seen, the TE polarization of incident waves is treated).

For a better understanding of the problem, it is convenient to clarify here the physical meaning of the amplitudes and phases appearing in Eqs. (1) and (2). In terms of holography, $\Omega_{10}\left(\Omega_{20}\right)$ is an amplitude of the object (reference) wave. In our case, describing the conversion situation in a monomode holocoupler, the wave emerging from the output face of the fiber and characterized by the Gaussian amplitude $\Omega_{10}$ and the cylindrical phase $p_{10}$ is taken as the object wave. The reference wave is an infinitely extended homogeneous plane wave. We choose the object- (reference-) wave-amplitude expression in terms of the product $\Psi_{10}$ and $\phi_{10}\left(\Psi_{20}\right.$ and $\left.\phi_{20}\right)$ in order to introduce a closer formal analogy between recording waves, characterized by Eqs. (1) and (2), and the reconstructed waves [see Eq. (15) below]. Without any special restrictions we can attribute the Gaussian (homogeneous-plane-wave) amplitude to $\phi_{10}\left(\phi_{20}\right)$ and consider $\Psi_{10}\left(\Psi_{20}\right)$ a constant normalization factor. If we bear in mind the holographic principle, the analogy between the formal aspect of Eqs. (1) and (2) and Eq. (15) becomes clearer. As a standard coupled-wave theory predicts, after the illumination of the hologram by the reference wave $\mathbf{E}_{2}$ that is similar to $\mathbf{E}_{20}$, the two waves that are closely related to those used for hologram construction appear inside the volume medium. If Bragg's condition is valid, the amplitude $\phi_{1}\left(\phi_{2}\right)$ is assumed to be close to $\phi_{10}\left(\phi_{20}\right)$. The factor $\Psi_{1}\left(\Psi_{2}\right)$, as opposed to the constant normalization factor $\Psi_{10}\left(\Psi_{20}\right)$, comprises the diffraction effects that are due to the multiple-scattering process in the modulated media, and it describes the spatial evolution of the diffracted amplitude of the field $\mathbf{E}_{1}\left(\mathbf{E}_{2}\right)$ as it propagates in the hologram. The functions $p_{10}$ and $p_{20}$ represent cylindrical and planewaves phases, respectively.

Considering the assumption made about $\Psi_{10}, \Psi_{20}, \phi_{10}$, $\phi_{20}, p_{10}$, and $p_{20}$ and applying normalization conditions for both incident waves, we can express the amplitudes and the phases as follows:

$$
\begin{aligned}
& \phi_{10}=\exp \left[-y^{2} /\left(2 \tau^{2}\right)\right], \\
& \phi_{20}=\Psi_{10}=\Psi_{20}=1, \\
& p_{10}=\rho=\left(x^{2}+y^{2}\right)^{1 / 2}, \\
& p_{20}=x,
\end{aligned}
$$

where $\tau$ is a parameter of the Gaussian beam. ${ }^{19}$
Assuming mutual coherence between the reference and the object waves in the recording area, uniform spatialfrequency response of the medium, and absence of a selfdeveloping mechanism in the media as well as in the linear recording process, the permittivity function in the area in which the interference occurs will have (after the developing process) the form

$$
\varepsilon_{r}=\varepsilon_{r 0}+\varepsilon_{r i}+\kappa\left|\mathbf{E}_{10}+\mathbf{E}_{20}\right|^{2},
$$

where $\varepsilon_{r i}=\varepsilon_{r i}{ }^{\prime}-j \varepsilon_{r i}{ }^{\prime \prime}$ is the change in the average value of the permittivity induced during the developing process and $\kappa=|\kappa| \exp (j \vartheta)$ is generally the complex constant that includes the sensitivity of the medium. As was assumed above, the complex constant has a constant value over the large interval of spatial frequencies and intensities.

Substituting Eqs. (1) and (2) into relation (7), the obtained expressions for $\varepsilon_{r}, \varepsilon_{n}{ }^{\prime}, \varepsilon_{r 1}{ }^{\prime}, \varepsilon_{n 1}{ }^{\prime \prime}$, and $\varepsilon_{r 1}{ }^{\prime \prime}$ are in the following form:

$$
\begin{aligned}
\varepsilon_{r}= & \varepsilon_{r 0}{ }^{\prime}+\varepsilon_{r i}^{\prime}-j\left(\varepsilon_{r 0}{ }^{\prime \prime}+\varepsilon_{r i}{ }^{\prime \prime}\right)+\left(\varepsilon_{n}^{\prime}-j \varepsilon_{n}^{\prime \prime}\right) \\
& +\left(\varepsilon_{r 1}{ }^{\prime}-j \varepsilon_{r 1}{ }^{\prime \prime}\right) \phi_{10} \phi_{20} \exp \left[-\alpha_{0}\left(p_{10}+p_{20}\right)\right] \\
& \times \cos \left[\beta_{0}\left(p_{20}-p_{10}\right)\right], \\
\varepsilon_{n}^{\prime}= & \operatorname{Re}(\kappa) \sum_{i=1}^{2}\left(\phi_{i 0} \Psi_{i 0}\right)^{2} \exp \left(-2 p_{i 0} \alpha_{0}\right), \\
\varepsilon_{r 1}{ }^{\prime}= & 2 \operatorname{Re}(\kappa) \Psi_{10} \Psi_{20}, \\
\varepsilon_{n}^{\prime \prime}= & -\varepsilon_{n}^{\prime} \arctan (\vartheta), \\
\varepsilon_{r 1}{ }^{\prime \prime}= & -\varepsilon_{r 1}{ }^{\prime} \arctan (\vartheta) .
\end{aligned}
$$

\section{Reconstruction Process}

For a nonmagnetic inhomogeneous linear medium with a permittivity given by relation (8), the wave equation takes the form ${ }^{20}$

$$
\nabla^{2} \mathbf{E}+\frac{\varepsilon_{r}}{c^{2}} \frac{\partial^{2} \mathbf{E}}{\partial t^{2}}+\nabla\left(\nabla \log \varepsilon_{r} \cdot \mathbf{E}\right)=0 .
$$

For simplicity we consider the situation of propagation of the monochromatic field, with angular frequency $\omega$ and TE polarization, in an inhomogeneous medium with its grating vector lying in the plane of incidence. Then the third term on the left-hand side of Eq. (13) vanishes, and the wave equation can be rewritten in the form

$$
\nabla^{2} \mathbf{E}(x, y)+\varepsilon_{r} \frac{\omega^{2}}{c^{2}} \mathbf{E}(x, y)=0 .
$$

Assuming that the conditions for the Bragg regime are fulfilled (meaning that the hologram is thick enough to justify the two-mode approximation) and that both the amplitude and the phase functions of the reconstructed wave are closed to those related to some of the original recording waves, we can expect a solution for the shaded area B in Fig. 1 according to the coupled-wave theory, in the following form:

$$
\begin{aligned}
\mathbf{E}(x, y) & =\mathbf{E}_{1}(x, y)+\mathbf{E}_{2}(x, y) \\
& =\sum_{i=1}^{2} \Psi_{i}(x, y) \phi_{i}(x, y) \exp \left[-\gamma p_{i}(x, y)\right] \hat{u}_{z} \\
& =\sum_{i} \Omega_{i}(x, y) \exp \left[-\gamma p_{i}(x, y)\right] \hat{u}_{z},
\end{aligned}
$$




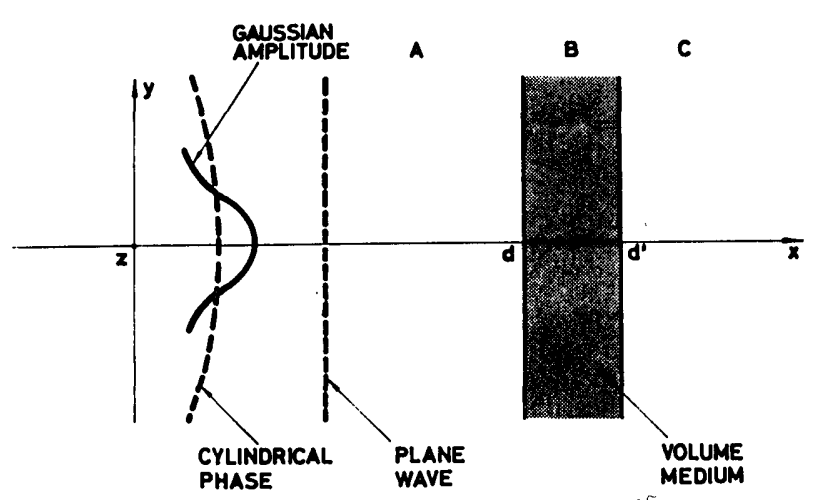

Fig. 1. Recording of a hologram by a Gaussian beam with a cylindrical phase and a plane wave in on-axis two-dimensional geometry. The Gaussian amplitude is the $\phi_{10}(x, y)$ function defined in Eq. (3), and the cylindrical phase is the $p_{10}$ term given in Eq. (5). Analogously, the plane-wave amplitude and the phase are defined in relations (4) and (6), respectively.

where $\gamma=j \beta+\alpha$ is a propagation constant in the modulated region $\mathrm{B} ; p_{i}$ is a phase function close to $p_{i 0} ; \phi_{i}$ is an amplitude function without any special restriction, but it is consistent to assume that $\phi_{i}$ is close to $\phi_{i 0} ; \Psi_{i}$ is an amplitude factor comprising diffraction effects of the modulated medium; and $\Omega_{i}=\Psi_{i} \phi_{i}$. Now wave equation (14) will be solved for a permittivity function given by Eq. (8); the solution anticipates the solution in the form of Eq. (15). One can expect that the spatial evolution of the amplitudes $\Psi_{i}$ is slower than the variation of the rapidly oscillating phase factors $\beta p_{i}$, in other words, that the diffracted amplitudes do not vary appreciably in the wavelength-dimension area. Substituting Eq. (15) into wave equation (14) and employing the above-mentioned assumption of slowly varying amplitudes (which requires mathematically that the terms $\nabla \phi_{i} \cdot \nabla \Psi_{i}$ and $\nabla^{2} \Psi_{i}$ vanish), we obtain the following equation:

$$
\begin{gathered}
\sum_{i=1}^{2} \exp \left(-\gamma p_{i}\right)\left(\Psi _ { i } \left\{\phi_{i} \gamma^{2}\left(\nabla p_{i}\right)^{2}-\gamma \phi_{i} \nabla^{2} p_{i}-2 \gamma \nabla \phi_{i} \cdot \nabla p_{i}\right.\right. \\
+\nabla^{2} \phi_{i}+\beta^{2} \phi_{i}\left[1+\frac{\varepsilon_{n}^{\prime}}{\varepsilon_{r}^{\prime}}-j\left(\frac{\varepsilon_{r}^{\prime \prime}}{\varepsilon_{r}^{\prime}}+\frac{\varepsilon_{n}^{\prime \prime}}{\varepsilon_{r}^{\prime}}\right)+\phi_{10} \phi_{20}\right. \\
\times \exp \left[-\alpha_{0}\left(p_{10}+p_{20}\right)\right] \cos \left[j \beta_{0}\left(p_{10}-p_{20}\right)\right] \\
\left.\left.\left.\times\left(\frac{\varepsilon_{r 1}{ }^{\prime}}{\varepsilon_{r}^{\prime}}-j \frac{\varepsilon_{r 1}{ }^{\prime \prime}}{\varepsilon_{r}^{\prime}}\right)\right]\right\}-2 \gamma \phi_{i} \nabla \Psi_{i} \cdot \nabla p_{i}\right)=0,
\end{gathered}
$$

where $\varepsilon_{r}{ }^{\prime}=\varepsilon_{r 0}{ }^{\prime}+\varepsilon_{r i}{ }^{\prime}$ [see Eq. (8)].

This equation must be valid for arbitrary values of permittivities $\varepsilon_{n}{ }^{\prime}, \varepsilon_{n}{ }^{\prime \prime}, \varepsilon_{r 1}{ }^{\prime}$, and $\varepsilon_{r 1}{ }^{\prime \prime}$; hence for a particular case without any modulation, when indeed the amplitudes $\Psi_{i}$ remain constant during a propagation, the coefficients at $\Psi_{i}$ must vanish. ${ }^{18}$ Now, introducing the loss parameter $\Gamma$ and the off-Bragg parameter $B$ with relations

$$
\begin{aligned}
& \Gamma=\alpha\left(p_{2}-p_{1}\right), \\
& B=\beta_{0}\left(p_{10}-p_{20}\right)-\beta\left(p_{1}-p_{2}\right),
\end{aligned}
$$

we easily obtain a system of the following coupled equations:

$$
\begin{aligned}
\nabla \Psi_{i} & \cdot \nabla p_{i}-\frac{\beta^{2}\left(\varepsilon_{n}{ }^{\prime}-j \varepsilon_{n}{ }^{\prime \prime}\right)}{2 \gamma \varepsilon_{r}{ }^{\prime}} \Psi_{i} \\
& -\frac{\beta^{2} \phi_{i 0} \phi_{k 0} \phi_{k}\left(\varepsilon_{r 1}{ }^{\prime}-j \varepsilon_{r 1}{ }^{\prime \prime}\right) \exp \left[-\alpha_{0}\left(p_{i 0}+p_{k 0}\right)\right]}{4 \varepsilon_{r}^{\prime} \gamma \phi_{i} \exp \left[(-1)^{k}(\Gamma+j B)\right]} \Psi_{k}=0,
\end{aligned}
$$

where $i, k=1,2$ and $i \neq k$.

The simplest concept then $\Gamma \approx 0$ is treated now. As can be seen from Eq. (17), the physical situation related to this condition is obviously a low-absorption or an on-axis geometry. ${ }^{21}$ Moreover, if an on-Bragg reconstruction process occurs $(B \approx 0)$, the propagation constant and the phase functions of the reconstructed waves are equivalent to those used for recording, and hence the following relations are valid:

$$
\begin{aligned}
p_{1} & =\rho=\left(x^{2}+y^{2}\right)^{1 / 2}, \\
p_{2} & =x, \\
\beta & =\beta_{0} .
\end{aligned}
$$

Now, ignoring the change of the average value of permittivity in relation (8) [as seen from Eq. (19), it is physically equivalent to ignoring the power transfer from a mode to itself], we can simplify coupled equations (19) to the form

$$
\begin{aligned}
& \nabla \Psi_{1} \cdot \nabla p_{1}+j k \phi_{2} \phi_{20} \Psi_{2}=0, \\
& \nabla \Psi_{2} \cdot \nabla p_{2}+j k \phi_{1} \phi_{10} \Psi_{1}=0,
\end{aligned}
$$

where

$$
k=\beta\left(\varepsilon_{r 1}{ }^{\prime}-j \varepsilon_{r 1}{ }^{\prime \prime}\right) /\left(4 \varepsilon_{r}{ }^{\prime}\right)
$$

is a coupling parameter.

Introducing the new variables $\nu$ and $\mu$ with the properties

$$
\begin{aligned}
& \nabla \nu \cdot \nabla p_{1}=0, \\
& \nabla \mu \cdot \nabla p_{2}=0,
\end{aligned}
$$

or, in other words, which are tangential to the co-phasal surfaces of the two diffraction modes (see Appendix A) and considering that

$$
\nabla=\nabla \nu \frac{\partial}{\partial \nu}+\nabla \mu \frac{\partial}{\partial \mu}
$$

we obtain coupled equations (23) and (24) in scalar form:

$$
\begin{aligned}
& \nabla \mu \cdot \nabla p_{1} \frac{\partial \Psi_{1}}{\partial \mu}=-j k \phi_{20} \phi_{2} \Psi_{2}, \\
& \nabla \nu \cdot \nabla p_{2} \frac{\partial \Psi_{2}}{\partial \nu}=-j k \phi_{10} \phi_{1} \Psi_{1} .
\end{aligned}
$$

In Appendix A the solutions of Eqs. (26) and (27) are shown to have the forms $\nu=h_{1}(y / x)$ and $\mu=h_{2}(y)$, where $h_{1}$ and $h_{2}$ are arbitrary functions. With respect to the symmetry of our geometrical configuration, it is favorable to choose (see Fig. 1)

$$
\begin{aligned}
\nu & =\arctan (y / x), \\
\mu & =y .
\end{aligned}
$$


Elimination of $\Psi_{2}$ in Eqs. (28) and (29) yields directly that

$$
\frac{k^{2} \phi_{10} \phi_{1} \phi_{20} \phi_{2}}{\nabla \nu \cdot \nabla p_{2}} \Psi_{1}=-\frac{\partial}{\partial \nu}\left(\nabla \mu \cdot \nabla p_{1}\right) \frac{\partial \Psi_{1}}{\partial \mu}-\nabla \mu \cdot \nabla p_{1} \frac{\partial^{2} \Psi_{1}}{\partial \nu \partial \mu} .
$$

Equation (32) is a second-order partial differential equation with nonconstant coefficients. In general, it is possible (as mentioned in the Introduction) to solve it by the generalized series method so that the fulfillment of the boundary-value requirements is inherent in the first member of the series, and the other members can be found by the iterative method. However, the proposed method converges very slowly, and complications arising with the analytical expression of the higher-order terms of the series increase geometrically in our case.

It is possible to simplify Eq. (32) by accepting the physical argument that the transfer between modes $\Psi_{1}$ and $\Psi_{2}$ is accomplished during their propagation in a direction perpendicular to the $\operatorname{grad}(\mu)$ vector (along the $x$ axis in Fig. 1). This means that in the plane perpendicular to the direction of propagation, the amplitudes of both modes depend very slightly on the $\mu$ coordinate. This assumption allows us to ignore the contribution of the first term on the right-hand side of the equation. If we express the scalar products of the gradients in Eq. (32) with respect to the definition of new variables (30) and (31) and use relations (3)-(6), it follows that a partial differential equation for the mode $\Psi_{1}$ has the form

$$
-\frac{\sin ^{3} \nu}{k^{2} \mu} \exp \left(\mu^{2} / \tau^{2}\right) \frac{\partial^{2} \Psi_{1}}{\partial \nu \partial \mu}+\Psi_{1}=0
$$

Now, introducing new variables,

$$
\begin{aligned}
& u=-\frac{\cos \nu}{2 \sin ^{2} \nu}+\frac{1}{2} \ln \tan \left(\frac{\nu}{2}\right), \\
& v=\frac{k^{2} \tau^{2}}{2} \exp \left(-\mu^{2} / \tau^{2}\right),
\end{aligned}
$$

and applying them in Eq. (33), we can obtain the resulting equation,

$$
\frac{\partial^{2} \Psi_{1}}{\partial u \partial v}+\Psi_{1}=0
$$

It is possible to solve this equation by use of the Riemann method. The derivation of the solution for this type of partial differential equation can be found in Ref. 22 . Assuming that the necessary condition for the existence of the solution of Eq. (36),

$$
\Psi_{1}[f(v), v]=0,
$$

is valid, the solution has the form

$$
\begin{aligned}
\Psi_{1}(u, v)= & \int_{f^{-1}(u)}^{v}\left(\frac{\partial \Psi_{1}}{\partial v^{\prime}}\right)_{v^{\prime}-f^{-1}(u)} \\
& \times J_{0}\left(2\left\{\left(v-v^{\prime}\right)\left[u-f\left(v^{\prime}\right)\right]\right\}^{1 / 2}\right) \mathrm{d} v^{\prime},
\end{aligned}
$$

where $f(v)$ is a function defining the curve where the boundary-value conditions are given for the mode $\Psi_{1}$, $f^{-1}(u)$ is an inverse function related to $f(v), J_{0}$ is a zero- order Bessel function of the first kind, and $v^{\prime}$ is a dummy variable.

Fulfillment of the boundary-value conditions requires mathematically that

$$
\begin{aligned}
& \Psi_{1}[f(v), v]=0 \\
& \Psi_{2}[f(v), v]=1 .
\end{aligned}
$$

It can be seen that the fact of a zero amplitude of diffracted mode $\Psi_{1}$ on the border between the incident and the holographic medium expressed by Eq. (39) ensures the validity of basic condition (37) for the existence of the Riemann solution, Eq. (38).

Now, using the fact that at the input surface $u=f(v)$ boundary conditions (39) and (40) must be fulfilled, Eq. (28) with respect to relations (34) and (35) takes the form

$$
\left(\frac{\partial \Psi_{1}}{\partial v}\right)_{v=f^{-1}(u)}=\frac{j k \tau}{2 v \sin \nu(u)}\left(\ln \frac{k^{2} \tau^{2}}{2 v}\right)^{-1 / 2} .
$$

Substituting this relation into solution (38), we obtain

$$
\begin{aligned}
\Psi_{1}(u, v)= & \frac{j k \tau}{2 \sin \nu(u)} \int_{f^{-1}(u)}^{v}\left[v^{\prime}\left(\ln \frac{k^{2} \tau^{2}}{2 v^{\prime}}\right)^{1 / 2}\right] \\
& \times J_{0}\left(2\left\{\left(v-v^{\prime}\right)\left[u-f\left(v^{\prime}\right)\right]\right\}^{1 / 2}\right) \mathrm{d} v^{\prime} .
\end{aligned}
$$

This is the final analytical integral expression of the - first mode of diffraction, assuming that the reconstructing wave is a homogeneous plane wave. ${ }^{23}$

Now, in order to obtain a solution for the amplitude $\Psi_{2}$, one cannot follow a way analogous to that for the amplitude $\Psi_{1}$. Eliminating $\Psi_{1}$ from Eqs. (28) and (29), one could obtain an equation similar to Eq. (36); however, the necessary condition for the existence of a Riemann-type solution is now $\Psi_{2}[f(v), v]=0$. This leads to an unreal physical situation, as the boundary-value condition requires that $\Psi_{2}[f(v), v]=1$. Nevertheless, to solve the problem one can directly integrate Eq. (29), which results in the following equation:

$$
\Psi_{2}=j \frac{2 v}{k \tau}\left(\ln \frac{k^{2} \tau^{2}}{2 v}\right)^{1 / 2} \int^{u} \frac{\Psi_{1}\left(u^{\prime}, v\right)}{\sin ^{2} \nu(u)} \frac{\mathrm{d} \nu}{\mathrm{d} u^{\prime}} \mathrm{d} u^{\prime}+C .
$$

Expressing $d \nu / d u^{\prime}$ in the integrand on the right-hand side of Eq. (43) with respect to relation (34), applying condition (40) to determine the integration constant $C$, and substituting $\Psi_{1}\left(u^{\prime}, v\right)$ according to Eq. (42), we can rewrite solution (43) in the resulting form:

$$
\begin{aligned}
\Psi_{2}(u, v)= & 1-v\left(\ln \frac{k^{2} \tau^{2}}{2 v}\right)^{1 / 2} \int_{f(v)}^{u} \int_{f^{-1}\left(u^{\prime}\right)}^{v} J_{0}\left(2 \left\{\left(v-v^{\prime}\right)\right.\right. \\
& \left.\left.\times\left[u^{\prime}-f\left(v^{\prime}\right)\right]\right\}^{1 / 2}\right)\left(v^{\prime}\left(\ln \frac{k^{2} \tau^{2}}{2 v^{\prime}}\right)^{1 / 2}\right. \\
& \times\left\{1-\frac{\sin ^{2} \nu\left(u^{\prime}\right)}{2}+\frac{\sin ^{3} \nu\left(u^{\prime}\right)}{4+\nu^{2}\left(u^{\prime}\right)}\right. \\
& \left.\left.\times\left[\frac{1+\cos \nu\left(u^{\prime}\right)}{1-\cos \nu\left(u^{\prime}\right)}\right]^{1 / 2}\right\}\right)^{-1} \mathrm{~d} v^{\prime} \mathrm{d} u^{\prime} .
\end{aligned}
$$

It can be seen that condition (40) is accomplished for the border area $[f(v)=u]$. In other words, the energy is to- 


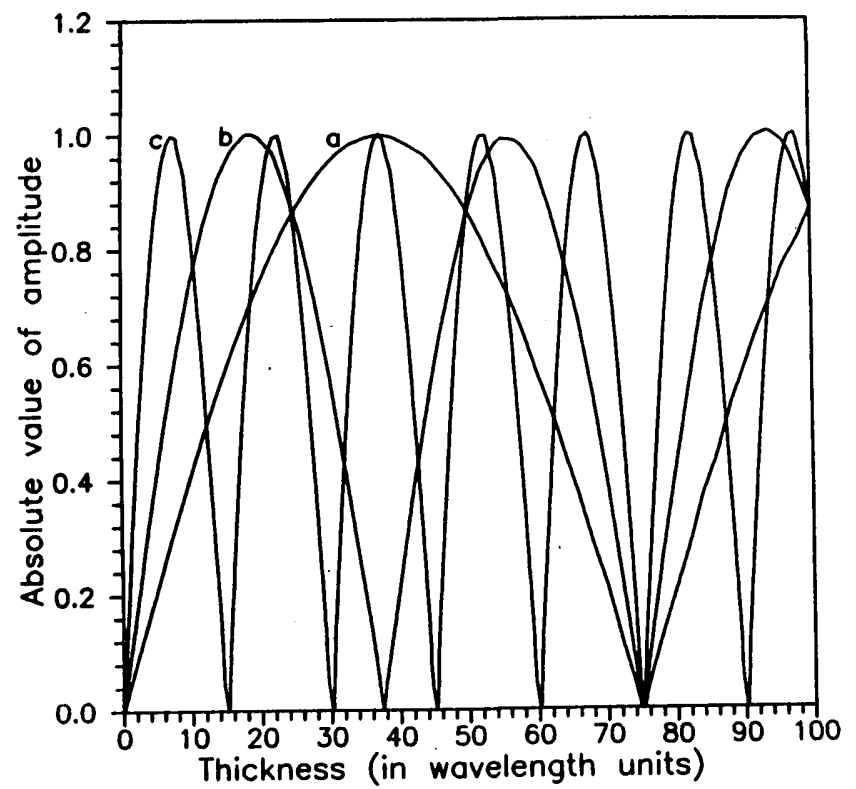

Fig. 2. Absolute value of the -first diffraction-mode amplitude $\Omega_{1}$ as a function of holographic-medium thickness $T$ for a near-axis point and for various values of parameter $\varepsilon_{r 1}{ }^{\prime} . T$ is in wavelength $(\lambda)$ units. Numerical parameters: $y=10^{-4} \mathrm{~m}$, $\varepsilon_{r}{ }^{\prime}=2.25, \tau=0.01 \mathrm{~m}, \lambda=0.5 \mu \mathrm{m}, d=0.1 \mathrm{~m}$. Values of parameter $\varepsilon_{r 1}^{\prime}$ : curve a, 0.01; curve b, 0.02; curve c, 0.05 .

tally carried by the reconstructed wave at the beginning of the mutual conversion process.

\section{NUMERICAL RESULTS AND DISCUSSION}

Figure 2 presents the evolution of the absolute value of the -first diffraction-mode amplitude $\Omega_{1}=\phi_{1} \Psi_{1}$ versus the thickness $T$ of the stratified medium (as can be seen in Fig. $\left.1, T=d^{\prime}-d\right)$ for a near-axis point $\left(y=10^{-4} \mathrm{~m}\right)$ and for various values of the parameter $\varepsilon_{r 1}{ }^{\prime}$. The fiberholographic-medium separation distance $d$ is chosen sufficiently large to ensure the cylindrical character of the phase of the Gaussian beam at the input plane $x=d(d=$ $0.1 \mathrm{~m}$ ). It can be clearly seen that decreasing $\varepsilon_{r 1}{ }^{\prime}$ causes a decrease in oscillation amplitude of function $\varepsilon_{r}$ and, obviously, in the value of the coupling parameter $k$ [see Eq. (25)]. The coupling between the two propagating modes therefore has less strength. In other words, the oscillation frequency of both amplitudes $\left(\Omega_{1}\right.$ and $\left.\Omega_{2}\right)$ decreases.

It can be seen that total energy transfer occurs between the modes, and hence high-efficiency coupling is accomplished. The proper selection of the output plane $x=d^{\prime}$ to ensure condition $\left|\Omega_{1}\right|=1$ depends on $\varepsilon_{r 1}$ ' (hence on the exposition energy), and this selection is crucial. In practical design, where it is extraordinarily difficult to control the holographic emulsion thickness with the accuracy required for optimal output-plane selection, the easiest way to achieve maximum fidelity of the reconstruction is to vary the energy of the exposure and hence the modulation of an exposed region.

In Fig. 3 an analogous dependence of $\left|\Omega_{1}\right|$ on thickness $T$ of the medium is presented with the $y$ coordinate as a parameter. The oscillation frequency decreases with the distance from the $x$ axis because the marginal parts of the hologram are less modulated than the central ones [see
Eqs. (8) and (3)]. However, it is interesting that for a relatively large distance from the central zone $(y>\tau)$, it is possible to achieve amplitudes equivalent to those for the near-axis area. Unfortunately, as can be seen in Fig. 4, this leads to the split of the diffracted beam and hence to a decrease in reconstruction fidelity.

A continuous splitting of the -first diffraction mode as it propagates in the modulated region $B$ is obvious from

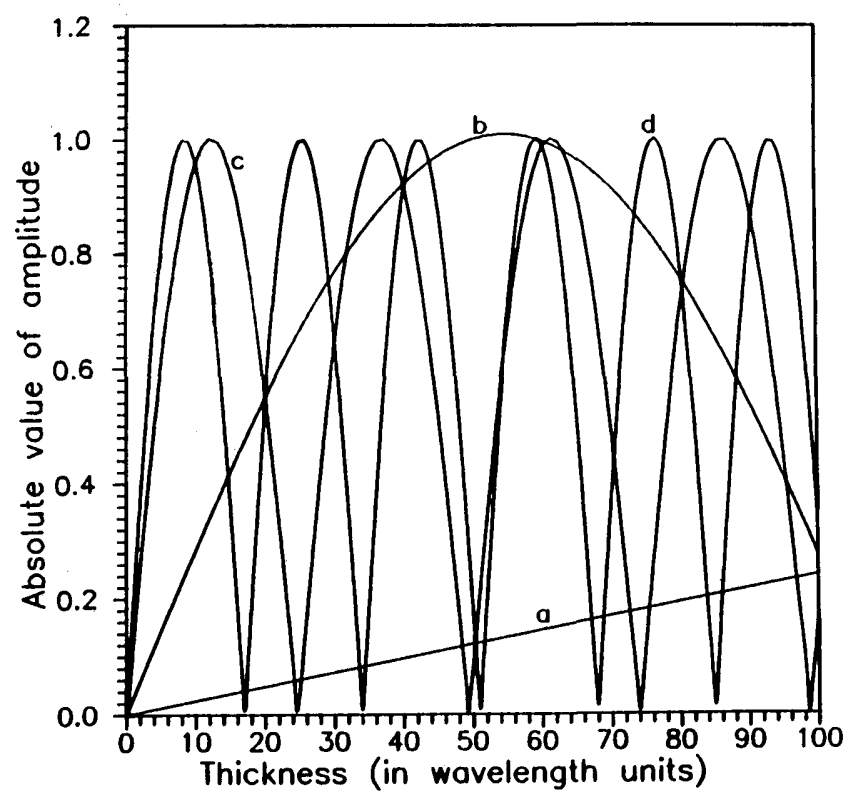

Fig. 3. Absolute value of the -first diffraction-mode amplitude $\Omega_{1}$ as a function of holographical medium thickness $T$ for various off-axis points. $T$ is in wavelength $(\lambda)$ units. Numerical parameters: $\varepsilon_{r 1}{ }^{\prime}=0.05, \varepsilon_{r}{ }^{\prime}=2.25, \tau=0.01 \mathrm{~m}, \lambda=0.5 \mu \mathrm{m}$, $d=0.1 \mathrm{~m}$. Values of parameter $y$ : curve a, $0.005 \mathrm{~m}$; curve $\mathrm{b}$, $0.01 \mathrm{~m}$; curve c, $0.02 \mathrm{~m}$; curve d, $0.03 \mathrm{~m}$.

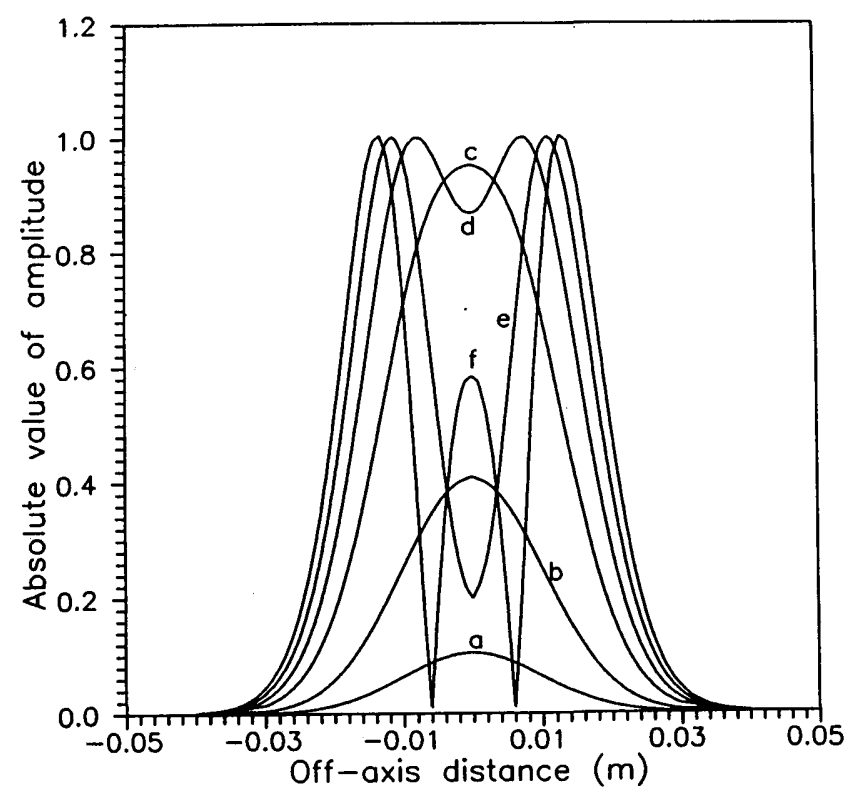

Fig. 4. Transversal distribution of the absolute value of amplitude $\Omega_{1}$ at the output plane $x=d^{\prime}$ of the hologram for various values of volume-medium thickness. Numerical parameters: $\varepsilon_{r 1}{ }^{\prime}=0.05, \varepsilon_{r}{ }^{\prime}=2.25, \tau=0.01 \mathrm{~m}, \lambda=0.5 \mu \mathrm{m}, d=0.1 \mathrm{~m}$. Values of parameter $T$ : curve a, $0.5 \lambda$; curve $b, 2 \lambda$; curve c, $6 \lambda$; curve $d$, $10 \lambda$; curve e, $14 \lambda$; curve $f, 18 \lambda$. 


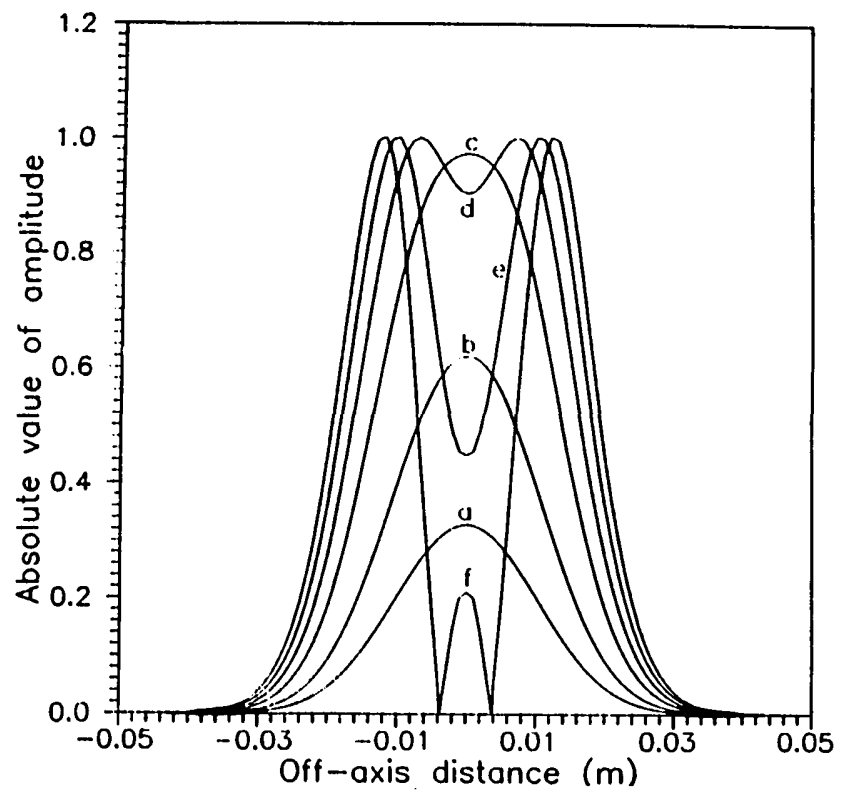

Fig. 5. Transversal distribution of the absolute value of amplitude $\Omega_{1}$ at the output plane $x=d^{\prime}$ of the hologram for various values of parameter $\varepsilon_{r 1}^{\prime}$. Numerical parameters: $\varepsilon_{r}^{\prime}=2.25$, $\tau=0.01 \mathrm{~m}, \lambda=0.5 \mu \mathrm{m}, d=0.1 \mathrm{~m}, T=8 \lambda$. Values of parameter $\varepsilon_{r 1}{ }^{\prime}:$ curve a, 0.01 ; curve b, 0.02; curve c, 0.04; curve $d, 0.06$; curve e, 0.08 ; curve $f, 0.1$.

Fig. 4, which presents the dependence of $\left|\Omega_{1}\right|$ on the transversal coordinate $y$. As one can see, theoretical diffraction efficiency close to 1 can be achieved with an appropriate selection of the output plane $x=d^{\prime}$. However, as the thickness $T$ of the modulated region increases above the optimal value, high efficiency and high fidelity can no longer be obtained simultaneously. The physical influence of the variation of $\varepsilon_{r 1}{ }^{\prime}$, shown in Fig. 5, is strictly analogous to those discussed above with respect to Figs. 2 and 3.

Certainly the beam-splitting effect has a much more complex physical nature than the one interpreted from Fig. 4. Our analysis was done under the approximation that the grating is strictly of a volume type in the entire area of the hologram, and hence the two-mode approximation is justified. Obviously, it is a rather strong condition when a Gaussian beam with a cylindrical-phase-to-planewave conversion is solved in on-axis geometry. In this case, the central zone of the grating can be characterized as being more similar to the Raman-Nath type than to the Bragg type. For these reasons, it is not feasible to anticipate an ideal diffraction efficiency for the central part of the hologram. Other problems that are associated with the overmodulation effect as a result of the limited dynamic range of the recording medium are important in practical considerations, but they are beyond the scope of this paper. It is of interest to mention that the splitting can be more effective when the recording process is accomplished with partially coherent light. If longitudinal coherence is poor, the marginal parts of the hologram will be less modulated, with an increased value in the average permittivity, and one can expect that the evolution of the splitting process will be faster in this case than for an ideal coherence situation. Certain analogies to this phenomenon can be found in the work of Juncos del Egido. ${ }^{24}$
In spite of the fact that our goal is to treat purely theoretical aspects of the coupling phenomena, it is convenient to introduce a brief practical insight into the possibilities of related applications.

The strongest restrictions of the model's applicability are obviously determined by the introduction of twodimensional space and on-axis geometry, which were chosen in order that we be able to treat the problem with sufficient mathematical rigor. Off-axis conversion between a Gaussian beam with spherical (and not cylindrical) phase and the plane wave, which describes the real monomode fiber-to-fiber coupling process, requires a rigorous three-dimensional approach, which is mathematically much more complex than the one presented. As far as we know, the problem has not been solved to date.

However, some rigorous applications of the model do exist. Two possible examples are (1) coupling to (or from) the planar waveguides and (2) a holographic collimator for the semiconductor lasers. Other possibilities are opened up in the spherical-to-cylindrical-to-plane-wave conver$\operatorname{sion}^{25}$ and in the design of holographic optical elements for astigmatism correction. The optical-fiber holocoupler more than likely remains the application easiest to test because of the possible use of optical fiber amplifiers to compensate the connection losses in the optical cross-bar interconnections. ${ }^{26}$

An attempt to introduce off-axis geometry into the analysis has been made; however, for analytical solutions to be obtained, a paraxial approximation is necessary. In a practical holographic optical element design, third-order aberrations disappear ${ }^{27}$ if an on-axis object wave is used. Obviously, for obtaining high diffraction efficiency (without the use of extremely thick recording emulsions), it is convenient to introduce a high-frequency carrier through an off-axis reference wave. In this case, new problems associated with polarization-dependent diffraction efficiency can arise when large Bragg angles are used, ${ }^{28}$ thus introducing additional optical noise in interconnectors that have circularly polarized light.

\section{CONCLUSIONS}

The theoretical model for a wave-front-conversion problem that arises in a monomode holographic coupler design was derived. The theory is based on the coupled-wave approach to the diffraction problem in a two-dimensional stratified dielectric medium. The numerical results show that the resulting integral solutions are consistent with the predictions of other models ${ }^{14,15}$ that treat similar conversion geometries.

The theory predicts that it is possible to achieve high diffraction efficiencies close to 1 . The splitting of the diffracted beam and hence a deterioration of reconstruction fidelity is also predicted. The importance of the proper selection of volume-medium thickness and the optimal expenditure of energy is found to be crucial.

The model can be used in the design and simulation of holographic couplers for monomode fibers, wave-front correctors, and any kind of holographic optical interconnection devices with geometries in which a Gaussian beam with cylindrical phase-to-plane-wave conversion appears. 


\section{APPENDIX A: SOLUTION OF THE DIFFERENTIAL EQUATION $\nabla u \cdot \nabla p=0$}

The equation that we are going to solve is a first-order linear partial differential equation. It can be written in the form

$$
u_{x} p_{x}+u_{y} p_{y}=0
$$

where the subscripts mean partial derivations.

After resolving the ordinary differential equation (characteristic equation)

$$
y^{\prime}=\frac{\mathrm{d} y}{\mathrm{~d} x}=\frac{p_{y}}{p_{x}}
$$

we can introduce new variables $\chi$ and $\sigma$ with the properties

$$
\begin{aligned}
\chi(x, y) & =\text { const } \\
\sigma & =y .
\end{aligned}
$$

Using Eqs. (A3) and (A4) we can see that

$$
\begin{aligned}
\chi_{x}+\chi_{y} y^{\prime} & =0 \\
u_{y} & =u_{\chi} \chi_{y}+u_{\sigma}, \\
u_{x} & =u_{\chi} \chi_{x} .
\end{aligned}
$$

After substitution of Eqs. (A6) and (A7) into Eq. (A1), we obtain

$$
u_{\chi}\left(p_{x} \chi_{x}+p_{y} \chi_{y}\right)+p_{y} u_{\sigma}=0
$$

With respect to Eqs. (A2) and (A5), it follows that

$$
p_{x} \chi_{x}+p_{y} \chi_{y}=p_{x}\left(\chi_{x}+\frac{p_{y}}{p_{x}} \chi_{y}\right)=p_{x}\left(\chi_{x}+y^{\prime} \chi_{y}\right)=0
$$

This means that, with respect to variables $\chi$ and $\sigma$, Eq. (A8) has the form

$$
p_{y} u_{\sigma}=0
$$

This equation must be fulfilled for arbitrary values of the function $p_{y}$. It means that the final solution has the form

$$
u=h(\chi)
$$

where $h$ is an arbitrary function of the argument $\chi$.

Now, solving Eq. (A1) for $p_{1}=\left(x^{2}+y^{2}\right)^{1 / 2}$ and then for $p_{2}=x$, one can easily find that $u_{1}=h_{1}(y / x)$ and $u_{2}=$ $h_{2}(y)$, where $h_{1}$ and $h_{2}$ are arbitrary functions of the arguments $y / x$ and $y$.

\section{ACKNOWLEDGMENTS}

When this research was initiated, P. Cheben was a fellow from the Czechoslovak government-Complutense University exchange program. The authors appreciate the financial support from these institutions.

*Present address, National Aerospace Institute (INTA), Space Instrumentation Laboratory, Carretera de Ajalvir Km. 4, Torrejón de Ardoz, Madrid 28050, Spain.

\section{REFERENCES AND NOTES}

1. B. Borrmann, "Über Extinktionsdiagramme von Quarz," Phys. Z. 42, 157-162 (1941).

2. P. St. J. Russell and L. Solymar, "The properties of holographic overlap gratings," Opt. Acta 26, 329-347 (1979).

3. P. St. J. Russell, "Reconstruction fidelity from volume holograms of finite width and variable index modulation," J. Opt. Soc. Am. 69, 496-503 (1979).

4. M. P. Jordan and L. Solymar, "On the properties of a finite reflection type volume hologram," Opt. Quantum Electron. 10, 503-507 (1978).

5. P. St. Russell, "Volume holographic finite-beam conversion with perfect fidelity," Opt. Acta 27, 997-1008 (1980).

6. P. St. J. Russell, L. Solymar, and M. P. Jordan, "Bormann-like effects in volume holography," presented at ICO-11, Madrid, 1978.

7. L. Solymar and M. P. Jordan, "Two-dimensional transmission type volume holograms for incident plane waves of arbitrary amplitude distribution," Opt. Quantum Electron. 9, 437-444 (1977).

8. R. S. Chu and T. Tamir, "Diffraction of Gaussian beams by periodically modulated media for incidence close to a Bragg angle," J. Opt. Soc. Am. 66, 1438-1440 (1976).

9. R. S. Chu and T. Tamir, "Bragg diffraction of Gaussian beams by periodically modulated media," J. Opt. Soc. Am. 66, 220-226 (1976).

10. V. M. Serdyuk, "The effect of self-diffraction of the writing waves on the diffraction efficiency of volume holograms," Sov. Phys. Tech. Phys. 34, 1097-1102 (1989).

11. S. Kusch and R. Güther, "Theorie der gekoppelten Wellen für Gauss-strahlen in der volumen Holographie," Exp. Tech. Phys. 25, 73-78 (1976).

12. W. E. Parry and L. Solymar, "A general solution for twodimensional volume holograms," Opt. Quantum Electron. 9, 527-531 (1977).

13. E. Guibelalde and M. L. Calvo, "A coupled wave analysis for on-axis holographic lenses in generalized coordinates," Opt. Commun. 59, 331-34 (1986).

14. L. Solymar and M. P. Jordan, "Analysis of cylindrical-to-plane wave conversion by volume holograms," Electron. Lett. 12, 143-144 (1976).

15. M. P. Jordan, L. Solymar, and P. St. J. Russell, "Wavefront conversion by volume holograms between cylindrical and plane waves," Microwaves Opt. Acoust. 2, 156-162 (1978).

16. M. L. Calvo and P. Cheben, "High-efficiency off-axis holographic coupler," Opt. Commun. 88, 22-26 (1992).

17. H. Kogelnik, "Coupled wave theory for thick hologram gratings," Bell Syst. Tech. J. 48, 2909-2947 (1969).

18. L. Solymar and D. J. Cooke, Volume Holography and Volume Gratings (Academic, Oxford, 1981).

19. In our case, $\tau$ depends mainly on the numerical aperture and the core diameter of the optical fiber as well as on the distance between the fiber output face and the input boundary of the hologram $\left(\tau \approx 10^{-2} \mathrm{~m}\right.$ can be considered in most practical cases).

20. M. Born and E. Wolf, Principles of Optics, 6th ed. (Pergamon, Oxford, 1980).

21. In the on-axis case, when $p_{1} \approx p_{2}$, there is obviously a negligible influence of the nonzero absorption $\alpha$ on the solution of Eq. (19). In addition, it is self-evident that a small value of parameter $B$ is determined by this geometry. However, on the other hand, it is necessary to ensure the accomplishment of conditions that characterize the Bragg regime (see Ref. 18, p. 129).

22. R. Courant and D. Hilbert, Methods of Mathematical Physics (Interscience, New York, 1953), Vol. 1, Chap. 5.

23. Rigorous holographic coupler design would require estimation of the case in which the reconstructed wave is a Gaussian beam. This is what really occurs in the first half of the fiber-to-fiber coupler if two-step conversion is accomplished (Gaussian-beam to plane-wave to Gaussian-beam conversion).

24. P. Juncos del Egido, "Diffraction of electromagnetic waves by a volume hologram treated as a periodic grating," Ph.D. dissertation (Universidad Complutense, Madrid, 1983).

25. Y. Defosse, Y. Renotte, and Y. Lion, "Calculation of diffraction efficiencies for spherical and cylindrical holographical 
lenses," in Holographical Optics III: Principles and Applications, G. M. Morris, ed., Proc. Soc. Photo-Opt. Instrum. Eng. 1507, 277-287 (1991).

26. D. G. Feitelson, L. Rudolph, and E. Schenfeld, "Limitations on optical free-space crossbar-like interconnection networks," in Optical Interconnections and Networks, H. Bartelt, ed., Proc. Soc. Photo-Opt. Instrum. Eng. 1281, 41-52 (1990).

27. D. Prongué and H. P. Herzig, "HOE for clock distribution in integrated circuits: experimental results," in Optical Inter- connections and Networks, H. Bartelt, ed., Proc. Soc. PhotoOpt. Instrum. Eng. 1281, 113-122 (1990).

28. J. S. Leggatt, G. R. Chamberlin, D. E. Sheat, and D. J. McCartney, "Holographically generated optical components for routeing and wavelength division multiplexing applications," Optical Interconnections and Networks, H. Bartelt, ed., Proc. Soc. Photo-Opt. Instrum. Eng. 1281, 227-243 (1990). 